\title{
Relationship between Gout and Diabetes Mellitus after Acute Pancreatitis: A Nationwide Cohort Study
}

\author{
Jaelim Cho (iD), Nicola Dalbeth (1D), and Maxim S. Petrov (iD)
}

\begin{abstract}
Objective. After acute pancreatitis, individuals often have low-grade inflammation, and subsequently develop metabolic sequelae such as post-pancreatitis diabetes mellitus (PPDM). Although numerous studies have investigated the relationship between gout and type 2 diabetes, little is known about the relationship between gout and PPDM. The aim was to investigate the associations between gout and PPDM.

Methods. Using nationwide pharmaceutical dispensing data linked to hospital discharge data in New Zealand, gout and PPDM were identified among individuals after first episode of acute pancreatitis between January 1, 2007, and December 31, 2015. Multivariable Cox regression analyses were conducted, adjusting for age, sex, ethnicity, social deprivation index, alcohol consumption, tobacco smoking, comorbidities, medications (glucocorticoids, statins, and estrogens), and characteristics of acute pancreatitis.

Results. A total of 10,117 individuals were included in the analysis of risk for gout and 9471 in the analysis of risk for PPDM. PPDM was significantly associated with a higher risk of gout in the overall cohort (adjusted HR 1.88, 95\% CI 1.15-3.06) and women (2.72, 95\% CI 1.31-5.65), but not in men $(1.42,95 \%$ CI $0.73-2.78)$. Preexisting gout was significantly associated with a higher risk of PPDM in the overall cohort (adjusted HR 1.58, 95\% CI 1.04-2.41) and women (2.66, 95\% CI 1.29-5.49), but not in men $(1.31,95 \%$ CI $0.78-2.20)$.

Conclusion. The relationship between gout and PPDM is bidirectional in the post-pancreatitis setting. A history of gout is a risk factor of PPDM, particularly in women. (First Release April 12020 ; J Rheumatol 2020;47:917-23; doi:10.3899/jrheum.190487)
\end{abstract}

Key Indexing Terms:

\section{GOUT DIABETES INFLAMMATION PANCREAS POPULATION-BASED STUDY}

Diabetes mellitus (DM) is a major cause of the global disease burden. The number of adults with DM in 2014 alone was 422 million $^{1}$, with the global economic burden estimated at US $\$ 1.3$ trillion $^{2}$. In the past decade, there has been growing evidence of the bidirectional association between type 2 diabetes and gout, as well as possible sex differences ${ }^{3,4,5,6,7,8}$. Several population-based studies demonstrated that gout is associated with a higher risk for type 2 diabetes in the general population ${ }^{5,8}$. These studies also showed that women have a significantly higher risk for type 2 diabetes compared with men (1.5 vs 1.2 times in the United Kingdom ${ }^{5} ; 2.0$ vs 1.6 times in Taiwan $^{8}$ ). By contrast, several population-based

From the School of Medicine, University of Auckland, Auckland, New Zealand.

This study was part of the Clinical and epidemiOlogical inveStigations in Metabolism, nutrition, and pancreatic diseaseS (COSMOS) program. COSMOS is supported, in part, by the Royal Society of New Zealand (Rutherford Discovery Fellowship to Associate Professor Max Petrov).

J. Cho, MD, MPH, School of Medicine, University of Auckland;

N. Dalbeth, MBChB, MD, School of Medicine, University of Auckland;

M.S. Petrov, MD, MPH, PhD, School of Medicine, University of Auckland.

Address correspondence to Associate Professor M.S. Petrov, Room

12.085A, Level 12, Auckland City Hospital, Auckland 1023, New Zealand.

E-mail:max.petrov@gmail.com

Accepted for publication September 24, 2019. studies demonstrated that type 2 diabetes is associated with a lower risk for gout ${ }^{6,7,9}$. A study from the United Kingdom reported that individuals with type 2 diabetes were at a $31 \%$ lower risk for gout compared with those without diabetes ${ }^{6}$. A study from Singapore demonstrated that type 2 diabetes was associated with a $23 \%$ lower risk for gout ${ }^{7}$. These studies also showed that the reduced risk for gout related to type 2 diabetes was significant only in men ${ }^{6,79}$.

Acute pancreatitis is the most common disease of the exocrine pancreas, with the incidence of 33.7 cases per 100,000 person-years worldwide ${ }^{10}$. While the old belief was that acute pancreatitis is a self-limiting disease, recent research has shown that individuals after acute pancreatitis often experience metabolic sequelae ${ }^{11}$. In particular, up to $40 \%$ of individuals after first episode of acute pancreatitis develop post-pancreatitis DM (PPDM) ${ }^{12}$. PPDM is the most common subtype of diabetes of the exocrine pancreas, which is in turn the second most common type of new-onset diabetes in adults ${ }^{13,14,15}$ and is known to lead to higher risks of mortality and hospitalization compared with type 2 diabetes ${ }^{16,17}$. Despite growing evidence of the association between type 2 diabetes and gout, to the best of our knowledge, no study to date has investigated the association between PPDM and gout in the post-pancreatitis setting. Given the association between type 2 diabetes and gout in

Personal non-commercial use only. The Journal of Rheumatology Copyright $($ C 2020. All rights reserved. 
previous literature, we hypothesized that PPDM is bidirectionally associated with gout in individuals after acute pancreatitis.

Our present study aimed to investigate the association between PPDM and gout among individuals following acute pancreatitis using a nationwide cohort.

\section{MATERIALS AND METHODS}

Data sources. The Ministry of Health Analytical Services (National Health Board, New Zealand) provided nationwide pharmaceutical dispensing data linked to hospital discharge and mortality data. The data included age, sex, ethnicity, area of residence, diagnostic codes, date of admission, and date and chemical name of subsidized prescriptions from primary to tertiary healthcare. All individuals were deidentified, so ethics approval was not required in accordance with the Ministry of Health guidelines.

Study cohort. The selection process is shown in Supplementary Figure 1 (available from the authors on request). Our present study followed people 20 years of age or older who were first admitted for acute pancreatitis [International Classification of Diseases, 10th revision (ICD-10), K85] as a primary diagnosis between January 1, 2006, and December 31, 2015. Date of the first admission for acute pancreatitis was set as index date. To investigate the association between PPDM and risk for gout, individuals without preexisting gout on the index date were included. In this analysis, the exposure was PPDM and the endpoint was the development of gout after the index date. To investigate the association between gout and risk for PPDM, individuals without preexisting diabetes on the index date were included. In this analysis, the exposure was the presence of gout prior to the index date and the endpoint was the development of PPDM after the index date.

Identification of gout and PPDM. Identification of gout was, in line with previous literature ${ }^{18}$, based on admission for gout (ICD-10, M10) or dispensing records of allopurinol or colchicine for gout, whichever came first. Allopurinol is often prescribed for patients with leukemia and lymphoma (ICD-10, C80-C96), so diagnosis of the diseases followed by dispensing records of allopurinol within 24 months was excluded from prescriptions of allopurinol for gout ${ }^{18}$. Individuals with diagnosis of gout or dispensing records of allopurinol or colchicine prior to or on the index date were considered as having preexisting gout.

PPDM was defined as admission for DM (ICD-10, E10, E11, and E13) and/or dispensing records of metformin 90 days after the index date (to rule out preexisting DM or stress-induced hyperglycemia), whichever came first ${ }^{11,19,20}$. We used only dispensing records of metformin, because the drug is the first-line therapy for type 2 diabetes. Severe liver disease (which is a contraindication for metformin) is rare in the post-pancreatitis setting ${ }^{21}$. Subjects with a diagnosis of DM or dispensing records of metformin prior to or within 90 days after the index date were considered to have preexisting diabetes.

Covariates. Alcohol consumption (ICD-10, F10) and tobacco smoking (Z720; Z8643; Z87.891) were defined as presence of the relevant diagnostic codes in any position between 1998 and 2015. Ethnicity was categorized as European, Māori, Pacific Islander, Asian, and others. Social deprivation index (based on area of residence) was classified into quartiles (the highest as the most deprived $)^{22}$. Charlson Comorbidity Index was calculated using ICD-10 codes in line with the previous literature ${ }^{23}$. Identification of individuals with previous hypertriglyceridemia was based on the relevant diagnostic code (ICD-10, E78.1) in any position on or prior to the index date. We also identified individuals who have dispensing records of the following medications on or prior to the index date: oral glucocorticoids, oral estrogen (for hormone replacement therapy), and statins.

Characteristics of acute pancreatitis included pancreatic cyst during followup, etiology, severity, and recurrence. Presence of pancreatic cyst during followup was defined as having the relevant diagnostic code (ICD-10, K86.2) in any position after the index date and until the end of followup. Gallstone acute pancreatitis was defined, in line with the previous literature ${ }^{22}$, as having diagnostic codes of gallstones and/or cholecystitis during the previous 3 years prior to or on the index date. Severe acute pancreatitis was defined, in line with the previous literature ${ }^{24}$, as having diagnostic codes of organ failure and/or infection during or within a 3-month period after the index date. Recurrent acute pancreatitis was defined as having 2 or more admissions for acute pancreatitis as a primary diagnosis. Hospital readmission within 30 days after the first episode of acute pancreatitis was not regarded as a new episode of acute pancreatitis ${ }^{22}$.

Statistical analysis. All analyses were performed using SAS version 9.4 (SAS Institute Inc.). T tests (for continuous variables) and chi-square tests (for categorical variables) were performed to examine differences in the characteristics between individuals with and without each endpoint. Cumulative incidence curves were created to compare proportions of individuals who developed gout (stratified by the presence of PPDM) and proportions of individuals who developed PPDM (stratified by presence of preexisting gout), with log-rank tests. To estimate the risk for gout related to PPDM and vice versa, multivariable Cox regression analyses were conducted. The assumption of proportional hazards over time was evaluated using graphical methods and fulfilled only in the analysis for the association between gout and risk for PPDM. Therefore, in the analysis for the association between PPDM and risk for gout, nonproportional Cox regression analysis was used, in which PPDM was treated as a time-dependent variable. In the time-dependent Cox regression analysis, individuals were classified as those without PPDM during the time from start of followup to their development of PPDM, from which point they were then reclassified as those with PPDM until the end of followup ${ }^{25}$. Model 1 was adjusted for age and sex. Model 2 was adjusted for age, sex, ethnicity, social deprivation index, alcohol consumption, tobacco smoking, and Charlson Comorbidity Index. In model 3, previous hypertriglyceridemia, oral glucocorticoid therapy, statin therapy, estrogen replacement therapy (only in women), and characteristics of acute pancreatitis (pancreatic cyst, etiology, severity, and recurrence) were adjusted for in addition to the covariates of model 2. The same analyses were conducted after stratification by sex. The risk was expressed as HR and $95 \% \mathrm{CI}$.

\section{RESULTS}

Characteristics of study individuals without preexisting gout. A total of 10,117 individuals were followed for $3.9 \pm 2.8$ years. Of these, $2.2 \%(n=219)$ of the individuals developed gout during followup (Table 1). The mean \pm SD ages were $62.1 \pm 16.5$ years and $55.8 \pm 19.2$ years in those with and without incident gout, respectively $(\mathrm{p}<0.001)$. Men accounted for $62.1 \%(\mathrm{n}=136)$ of those with incident gout and $45.7 \%(n=4525)$ of those without incident gout $(\mathrm{p}<0.001)$. Individuals who were most socially deprived (quartile 4) accounted for $36.5 \%(n=80)$ of those with incident gout and $26.5 \%(\mathrm{n}=2622)$ of those without incident gout $(p=0.003)$. Characteristics of the study individuals without preexisting gout stratified by PPDM status are presented in Supplementary Table 1 (available from the authors on request).

PPDM and risk of gout. Individuals with PPDM had a higher proportion of gout $(3.3 \%, \mathrm{n}=19$ out of 583) than those without PPDM (2.1\%, n = 200 out of 9534; Figure 1A; Supplementary Figure 2A, available from the authors on request). After adjusting for age and sex (model 1), individuals with PPDM were at a significantly higher risk for gout than those without PPDM (HR 2.20, 95\% CI 1.36-3.56; Table 2). After adding the other covariates to model 1 ,

Personal non-commercial use only. The Journal of Rheumatology Copyright $(\subset) 2020$. All rights reserved. 
Table 1. Characteristics of study subjects stratified by gout (outcome) status.

\begin{tabular}{|c|c|c|c|c|c|c|c|}
\hline \multirow{2}{*}{$\begin{array}{l}\text { Characteristics } \\
\text { Age, yrs, mean (SD) }\end{array}$} & \multicolumn{2}{|c|}{ Total, $\mathrm{n}=10,117$} & \multicolumn{2}{|c|}{$\begin{array}{l}\text { Without Incident } \\
\text { Gout, } \mathrm{n}=9898\end{array}$} & \multicolumn{2}{|c|}{$\begin{array}{l}\text { With Incident } \\
\text { Gout, } \mathrm{n}=219\end{array}$} & \multirow{2}{*}{$\begin{array}{c}\mathrm{p} \\
<0.001\end{array}$} \\
\hline & 55.9 & (19.1) & 55.8 & $(19.2)$ & 62.1 & $(16.5)$ & \\
\hline Ethnicity & & & & & & & $<0.001$ \\
\hline European & 7225 & $(71.4)$ & 7097 & $(71.7)$ & 128 & $(58.5)$ & \\
\hline Māori & 1429 & $(14.1)$ & 1382 & $(14.0)$ & 47 & $(21.5)$ & \\
\hline Others & 342 & (3.4) & 336 & (3.4) & 6 & (2.7) & \\
\hline Social deprivation index & & & & & & & 0.003 \\
\hline Quartile 1 & 2631 & $(26.0)$ & 2594 & $(26.2)$ & 37 & $(16.9)$ & \\
\hline Quartile 2 & 1811 & $(17.9)$ & 1774 & $(17.9)$ & 37 & $(16.9)$ & \\
\hline Quartile 3 & 2343 & $(23.2)$ & 2289 & $(23.1)$ & 54 & $(24.7)$ & \\
\hline Quartile 4 (most deprived) & 2702 & $(26.7)$ & 2622 & $(26.5)$ & 80 & $(36.5)$ & \\
\hline Hypertriglyceridemia & 44 & $(0.4)$ & 42 & $(0.4)$ & 2 & $(0.9)$ & 0.57 \\
\hline Oral glucocorticoid therapy & 2959 & $(29.3)$ & 2862 & $(28.9)$ & 97 & $(44.3)$ & $<0.001$ \\
\hline Statin therapy & 3106 & $(30.7)$ & 2999 & $(30.3)$ & 107 & $(48.9)$ & $<0.001$ \\
\hline Estrogen therapy (out of women) & 117 & $(2.1)$ & 115 & $(2.1)$ & 2 & $(0.9)$ & 0.02 \\
\hline Pancreatic cyst during followup & 13 & $(0.1)$ & 11 & $(0.1)$ & 2 & $(0.9)$ & 0.02 \\
\hline Gallstone acute pancreatitis & & & & & & & 0.089 \\
\hline Yes & 3053 & $(30.2)$ & 2975 & $(30.1)$ & 78 & $(35.6)$ & \\
\hline No & 7064 & $(69.8)$ & 6923 & $(69.9)$ & 141 & $(64.4)$ & \\
\hline Severe acute pancreatitis & & & & & & & 0.33 \\
\hline Yes & 1109 & $(11.0)$ & 1080 & $(10.9)$ & 29 & $(13.2)$ & \\
\hline No & 9008 & $(89.0)$ & 8818 & $(89.1)$ & 190 & $(86.8)$ & \\
\hline Recurrent acute pancreatitis & & & & & & & 0.34 \\
\hline
\end{tabular}

P values were from $t$ tests (for continuous variables) and chi square tests (for categorical variables) between individuals with and without incident gout. Values are $\mathrm{n}(\%)$ unless otherwise specified.

A

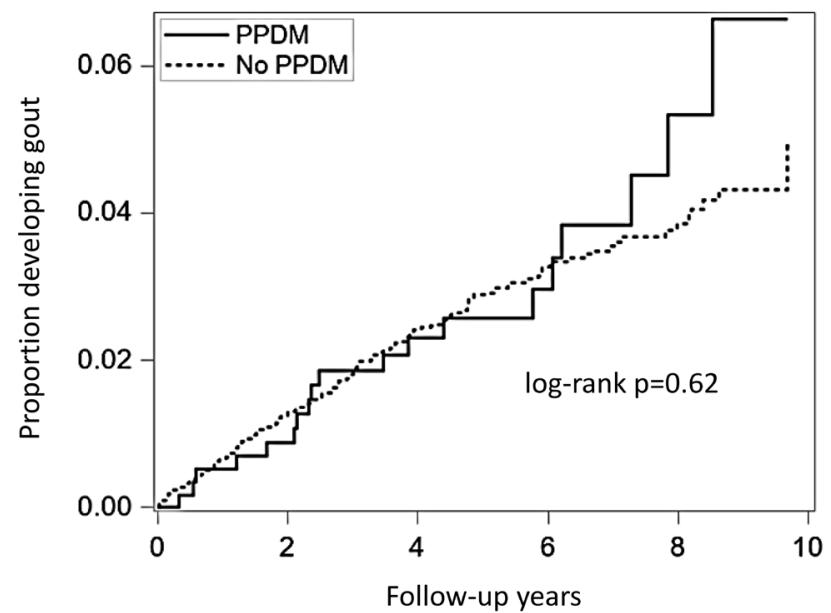

B

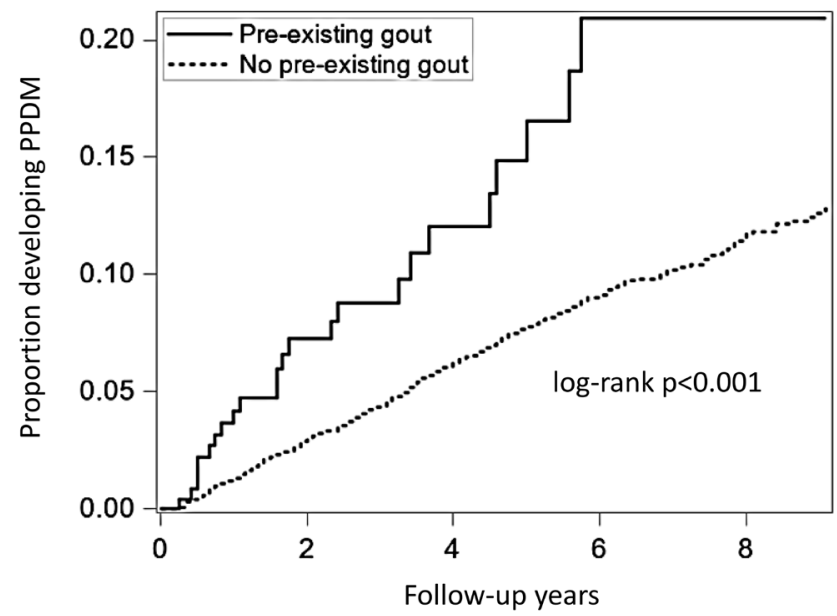

Figure 1. Cumulative incidence of (A) gout after acute pancreatitis stratified by the presence/absence of post-pancreatitis diabetes mellitus, and (B) post-pancreatitis diabetes mellitus stratified by the presence/absence of preexisting gout. PPDM: post-pancreatitis diabetes mellitus. 
Table 2. Association between post-pancreatitis diabetes mellitus and risk for gout after first episode of acute pancreatitis.

\begin{tabular}{ll}
\hline Variables & HR $(95 \% \mathrm{CI})$ \\
\hline Overall & \\
Model 1 & $2.20(1.36-3.56)$ \\
Model 2 & $1.88(1.16-3.05)$ \\
Model 3 & $1.88(1.15-3.06)$ \\
Men & \\
Model 1 & $1.63(0.85-3.15)$ \\
Model 2 & $1.39(0.72-2.68)$ \\
Model 3 & $1.42(0.73-2.78)$ \\
Women & \\
Model 1 & $3.57(1.76-7.24)$ \\
Model 2 & $3.01(1.47-6.20)$ \\
Model 3 & $2.72(1.31-5.65)$ \\
\hline
\end{tabular}

HR (95\% CI) was from time-dependent Cox regression models. Model 1: adjusted for age and sex (only in the overall analysis). Model 2: adjusted for the model 1 variables plus ethnicity, social deprivation index, smoking, alcohol consumption, and Charlson Comorbidity Index. Model 3: adjusted for the model 2 variables plus hypertriglyceridemia, oral glucocorticoid therapy, statin therapy, estrogen replacement therapy (women only), pancreatic cyst during followup, and etiology, severity, and recurrence of acute pancreatitis.

the association remained significant (HR 1.88, 95\% CI 1.16-3.05 in model 2; HR 1.88, 95\% CI 1.15-3.06 in model $3)$. In the analysis stratified by sex, the proportion of incident gout among individuals with PPDM was 3.3\% (10 out of 308) and 3.3\% (9 out of 275) in men and women, respectively. In the multivariable analyses, PPDM was significantly associated with a higher risk for gout in all the models among women only (Table 2).

Characteristics of study individuals without preexisting diabetes. A total of 9471 individuals were followed for $3.8 \pm 2.8$ years. Of these, $5.9 \%(n=558)$ of the individuals developed PPDM (Table 3 ). The mean \pm SD ages were $56.6 \pm 17.3$ years in individuals with PPDM and 56.0 \pm 19.5 years in those without PPDM $(\mathrm{p}=0.47)$. Men accounted for $54.8 \%(n=306)$ of those with PPDM and $47.1 \%$ $(\mathrm{n}=4200)$ of those without PPDM $(\mathrm{p}<0.001)$. The proportions of individuals with the highest social deprivation index were $33.7 \%(n=188)$ and $25.2 \%(n=2243)$ in those with and without PPDM, respectively $(\mathrm{p}<0.001)$. Characteristics of the study individuals without preexisting diabetes stratified by preexisting gout status are presented in Supplementary Table 2, (available from the authors on request).

Preexisting gout and risk for PPDM. People with preexisting gout had a higher proportion of PPDM $(9.1 \%$, $\mathrm{n}=24$ out of 265$)$ than those without preexisting gout $(5.8 \%$, $n=534$ out of 9206; Figure 1B; Supplementary Figure 2B, available from the authors on request). After adjusting for age and sex, subjects with preexisting gout had a significantly higher risk for PPDM than those without preexisting gout (HR 1.94, 95\% CI 1.28-2.93; Table 4). After adding the other covariates to model 1 , the association between preexisting gout and PPDM remained significant (HR 1.57, 95\% CI 1.03-2.38 in model 2; HR 1.58, 95\% CI 1.04-2.41 in model 3 ). In the analysis stratified by sex, the proportion of PPDM among individuals with preexisting gout was $8.6 \%$ (16 out of 186) and $10.1 \%$ (8 out of 79) in men and women, respectively. In the multivariable analyses, preexisting gout was significantly associated with a higher risk for PPDM in all the models only among women (Table 4).

\section{DISCUSSION}

To our knowledge, our present study is the first to investigate the bidirectional association between DM and gout following acute pancreatitis, a common inflammatory disease frequently accompanied by new-onset metabolic sequelae ${ }^{11}$. By using a nationwide database, selection bias was minimized. In addition, using pharmaceutical dispensing data covering primary to tertiary healthcare enabled us to reliably identify individuals with gout, who are often first diagnosed in the community. The main finding in our present study is that patients with PPDM were at a 1.9 times significantly higher risk for gout, whereas those with preexisting gout were at a 1.6 times significantly higher risk for PPDM in the overall cohort after adjusting for potential confounders. Further, women with PPDM were at a 2.7 times significantly higher risk for gout, and women with preexisting gout were at a 2.7 times significantly higher risk for PPDM.

Several population-based studies have investigated the association between type 2 diabetes and risk for gout $t^{6,7,9}$. The present study found a significant association between PPDM and a higher risk for gout in the overall cohort, which is somewhat inconsistent with the studies reporting the association between type 2 diabetes and the lower risk for gout ${ }^{6,7}$. We also found a significant association between PPDM and a higher risk for gout among women, whereas previous studies showed null association between type 2 diabetes and gout in women ${ }^{6,7}$. One of the reasons for the discordant findings might be the methodological differences in identifying individuals with gout. The 2 previous studies defined gout as diagnosis in either primary care only ${ }^{6}$ or self-report ${ }^{7}$, whereas the present study used dispensing data on urate-lowering agents (longterm treatment for gout) in primary to tertiary care as well as hospital admission data on gout. This approach may have enabled us to minimize the selection bias and recall bias. Another reason for the discrepancy may relate to the different study populations The previous studies targeted general populations, whereas our present study included individuals after acute pancreatitis only. Moreover, our cohort included more diverse ethnic populations (NZ European, Māori, Pacific, and Asian) compared with the previous studies. Given the known high heritability of serum urate levels (between $40-70 \%{ }^{26,27,28}$ ) and taking into account the role of gene-environment interactions in the risk of gout ${ }^{29}$, it is conceivable that certain ethnic groups in our study population might have been more

Personal non-commercial use only. The Journal of Rheumatology Copyright (c) 2020. All rights reserved. 
Table 3. Characteristics of study subjects stratified by PPDM (outcome) status.

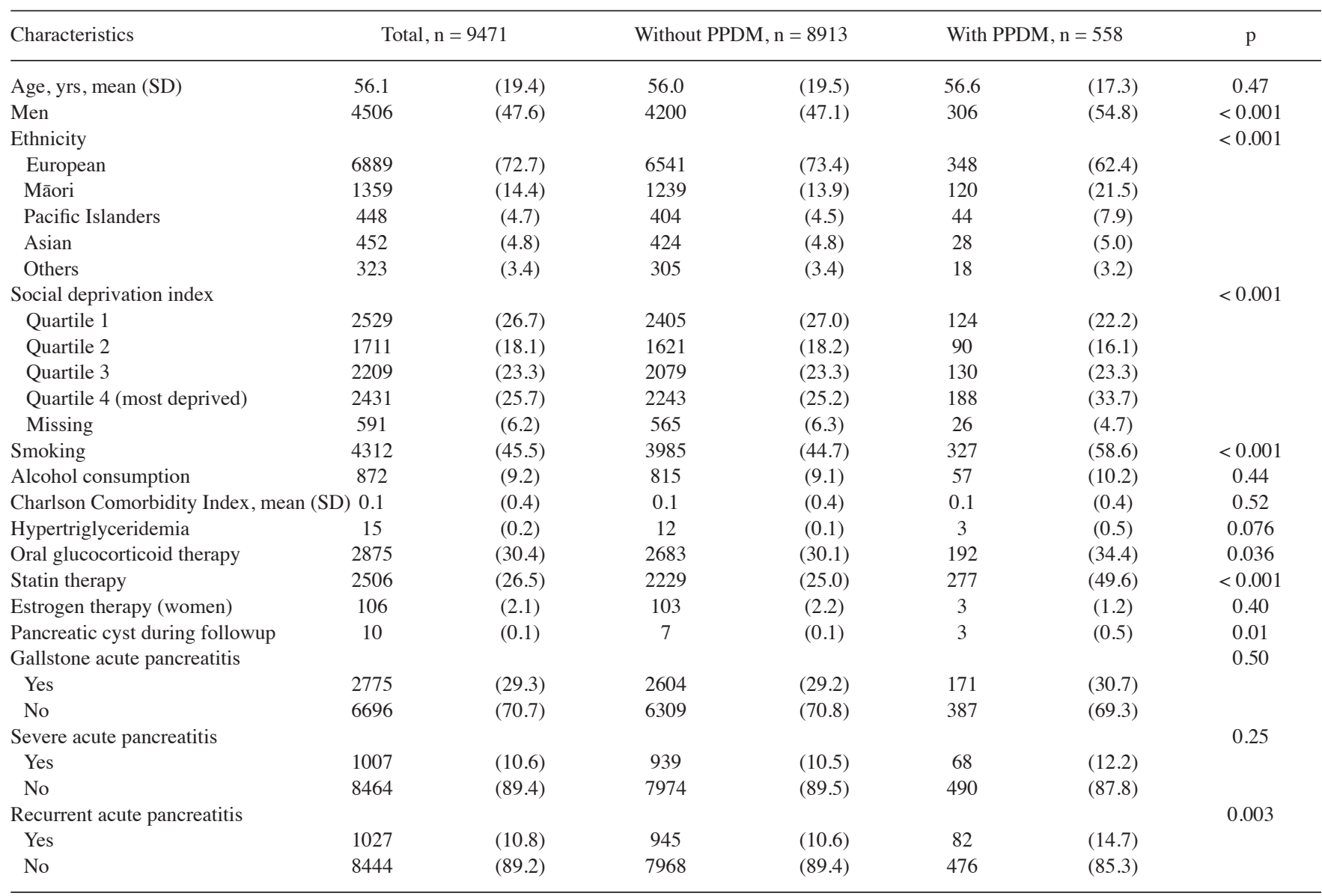

$\mathrm{P}$ values were from $\mathrm{t}$ tests (for continuous variables) and chi-square tests (for categorical variables) between individuals with and without PPDM. Values are n (\%) unless otherwise specified. PPDM: post-pancreatitis diabetes mellitus.

Table 4. Association between preexisting gout and risk for post-pancreatitis diabetes mellitus.

\begin{tabular}{ll}
\hline Variables & HR $(95 \%$ CI $)$ \\
\hline Overall & \\
Model 1 & $1.94(1.28-2.93)$ \\
Model 2 & $1.57(1.03-2.38)$ \\
Model 3 & $1.58(1.04-2.41)$ \\
Men & \\
Model 1 & $1.55(0.93-2.56)$ \\
Model 2 & $1.27(0.76-2.13)$ \\
Model 3 & $1.31(0.78-2.20)$ \\
Women & \\
Model 1 & $3.34(1.65-6.79)$ \\
Model 2 & $2.63(1.28-5.40)$ \\
Model 3 & $2.66(1.29-5.49)$ \\
\hline
\end{tabular}

HR $(95 \%$ CI) was from conventional Cox regression models. Model 1: adjusted for age and sex (only in the overall analysis). Model 2: adjusted for the model 1 variables plus ethnicity, social deprivation index, smoking, alcohol consumption, and Charlson Comorbidity Index. Model 3: adjusted for the model 2 variables plus hypertriglyceridemia, oral glucocorticoid therapy, statin therapy, estrogen replacement therapy (women), pancreatic cyst during followup, and etiology, severity, and recurrence of acute pancreatitis. predisposed to gout. Larger studies are warranted to elucidate genetic and ethnic variations in the association between PPDM and risk for gout. It is interesting to note that individuals with PPDM showed a higher cumulative incidence of gout compared with those without PPDM, particularly 6 years after first episode of acute pancreatitis (Figure 1A). This indicates that gout might be a late complication related to PPDM among individuals after the first episode of acute pancreatitis.

The association between gout and risk of type 2 diabetes has been investigated in general populations in the United States, United Kingdom, Taiwan, and Singapore ${ }^{3,5,7,8}$. In the present study, individuals with preexisting gout were at a significantly higher risk for PPDM in the overall cohort, which is consistent with the previous type 2 diabetes studies from the United Kingdom and Taiwan ${ }^{5,8}$. In addition, the association between gout and risk for PPDM was significant only in women in our present study, which is consistent with the previous studies showing stronger associations between gout and type 2 diabetes in women compared with men $^{5,8}$. This sex difference is supported by several studies 
in nondiabetic individuals that have shown that the positive association between serum urate and insulin resistance is stronger in women compared with men ${ }^{30,31}$. However, the association between serum urate levels (or urate metabolism) and insulin resistance has never been investigated in the post-pancreatitis setting, which now warrants a purposely designed study.

Our present study is the first ever to investigate the bidirectional relationship between PPDM and gout, to our knowledge; hence, we can only speculate on possible biological mechanisms linking PPDM and gout or abnormal glucose metabolism and hyperuricemia. One possible explanation might be altered glucose and lipid metabolism after acute pancreatitis, as our previous studies have demonstrated ${ }^{32,33,34,35,36}$. Specifically, upregulated inflammatory process induces lipolysis of adipose tissue in individuals with PPDM (but not in those without PPDM) and lipolysis of adipose tissue may raise the level of serum urate $^{37}$. Further, the pathophysiology of PPDM involves increased insulin resistance ${ }^{11,34,35}$, which was found to be associated with hyperuricemia in several clinical and animal studies $^{31,38,39}$. These convoluted mechanisms may have contributed to the bidirectional association between PPDM and gout.

There are several limitations to acknowledge. First, the identification of PPDM was based on ICD-10 codes only, because fasting blood glucose or glycated hemoglobin data were not available. Hence, the number of individuals with PPDM might have been underestimated. However, this would not have classified the study groups differentially. Second, data on serum urate were not available. We identified individuals with gout using dispensing data of urate-lowering medications, which means that individuals clinically diagnosed with gout were included ${ }^{18}$. Moreover, those who had a potential to receive urate-lowering medications owing to other causes (e.g., leukemia) were excluded in the identification of gout. This approach might have led to underestimation of gout. However, it is unlikely to have differentially affected the PPDM status of study subjects. Third, the present study did not consider dietary habits, a common risk factor for the development of gout. However, a 2018 metaanalysis demonstrated that dietary habits (using 63 food questionnaire items) contributed to serum urate levels much less than did genetic factors $(4.3 \%$ vs $23.9 \%$ of the variation in serum urate levels) ${ }^{40}$. Further, mounting evidence suggests that dietary habits (e.g., soft drink and alcohol consumption) interact with gene variants in developing hyperuricemia and gout $^{41,42,43,44,45}$. There is no population-based study considering the effect of gene-diet interactions on the association between DM and gout, which needs to be investigated in the future. Fourth, alcohol consumption was defined using ICD codes. We acknowledge that there might have been residual confounding by moderate to heavy alcohol consumption. For the same reason, it was not possible to reliably identify alcohol etiology of acute pancreatitis in our dataset. However, detailed information on alcohol consumption (e.g., amount, frequency, and type of drinks) is not recorded in most administrative databases. Last, data on body mass index were not available because anthropometric measurements were not recorded in the administrative databases used. Acquisition of these data could be beneficial for better understanding of mechanisms behind the association between PPDM and gout.

Our present study shows that there are significantly positive associations between PPDM and gout in the overall cohort and women, and the associations are bidirectional. These findings indicate that individuals with PPDM are at high risk for gout and individuals with preexisting gout are at high risk for PPDM, particularly women. This has implications for primary prevention of not only gout but also PPDM in women after acute pancreatitis. Given a paucity of evidence on the mechanism behind this relationship, clinical studies are now warranted to investigate serum urate levels and urate metabolism after acute pancreatitis. Multidisciplinary approaches to management of diabetes and gout after pancreatitis encompassing rheumatologists, endocrinologists, and gastroenterologists may prove beneficial in the future.

\section{REFERENCES}

1. NCD Risk Factor Collaboration (NCD-RisC). Worldwide trends in diabetes since 1980: a pooled analysis of 751 population-based studies with 4.4 million participants. Lancet 2016;387:1513-30.

2. Bommer C, Sagalova V, Heesemann E, Manne-Goehler J, Atun $\mathrm{R}$, Barnighausen T, et al. Global economic burden of diabetes in adults: projections from 2015 to 2030 . Diabetes Care 2018; 41:963-70

3. Choi HK, De Vera MA, Krishnan E. Gout and the risk of type 2 diabetes among men with a high cardiovascular risk profile. Rheumatology 2008;47:1567-70.

4. Kim SC, Liu J, Solomon DH. Risk of incident diabetes in patients with gout: a cohort study. Arthritis Rheumatol 2015;67:273-80.

5. Rho YH, Lu N, Peloquin CE, Man A, Zhu Y, Zhang Y, et al. Independent impact of gout on the risk of diabetes mellitus among women and men: a population-based, BMI-matched cohort study. Ann Rheum Dis 2016;75:91-5.

6. Rodriguez G, Soriano LC, Choi HK. Impact of diabetes against the future risk of developing gout. Ann Rheum Dis 2010;69:2090-4.

7. Pan A, Teng GG, Yuan JM, Koh WP. Bidirectional association between diabetes and gout: the Singapore Chinese Health study. Sci Rep 2016;6:25766.

8. Tung YC, Lee SS, Tsai WC, Lin GT, Chang HW, Tu HP. Association between gout and incident type 2 diabetes mellitus: a retrospective cohort study. Am J Med 2016;129:1219.e17-25.

9. Wijnands JM, van Durme CM, Driessen JH, Boonen A, Klop C, Leufkens B, et al. Individuals with type 2 diabetes mellitus are at an increased risk of gout but this is not due to diabetes: a population-based cohort study. Medicine 2015;94:e1358.

10. Xiao AY, Tan ML, Wu LM, Asrani VM, Windsor JA, Yadav D, et al Global incidence and mortality of pancreatic diseases: a systematic review, meta-analysis, and meta-regression of population-based cohort studies. Lancet Gastroenterol Hepatol 2016;1:45-55.

11. Petrov MS, Yadav D. Global epidemiology and holistic prevention of pancreatitis. Nat Rev Gastroenterol Hepatol 2019;16:175-84.

Personal non-commercial use only. The Journal of Rheumatology Copyright @ 2020 . All rights reserved. 
12. Das SL, Singh PP, Phillips AR, Murphy R, Windsor JA, Petrov MS. Newly diagnosed diabetes mellitus after acute pancreatitis: a systematic review and meta-analysis. Gut 2014;63:818-31.

13. Pendharkar SA, Mathew J, Zhao J, Windsor JA, Exeter DJ, Petrov MS. Ethnic and geographic variations in the incidence of pancreatitis and post-pancreatitis diabetes mellitus in New Zealand: a nationwide population-based study. N Z Med J 2017;130:55-68.

14. Woodmansey C, McGovern AP, McCullough KA, Whyte MB, Munro NM, Correa AC, et al. Incidence, demographics, and clinical characteristics of diabetes of the exocrine pancreas (type 3c): a retrospective cohort study. Diabetes Care 2017;40:1486-93.

15. Pendharkar SA, Mathew J, Petrov MS. Age- and sex-specific prevalence of diabetes associated with diseases of the exocrine pancreas: a population-based study. Dig Liver Dis 2017;49:540-4.

16. Cho J, Scragg R, Petrov MS. Risk of mortality and hospitalization after post-pancreatitis diabetes mellitus vs type 2 diabetes mellitus: a population-based matched cohort study. Am J Gastroenterol 2019;114:804-12.

17. Cho J, Scragg R, Pandol SJ, Goodarzi MO, Petrov MS. Antidiabetic medications and mortality risk in individuals with pancreatic cancer-related diabetes and postpancreatitis diabetes: a nationwide cohort study. Diabetes Care 2019;42:1675-83.

18. Winnard D, Wright C, Taylor WJ, Jackson G, Te Karu L, Gow PJ, et al. National prevalence of gout derived from administrative health data in Aotearoa New Zealand. Rheumatology 2012;51:901-9.

19. Petrov MS. Diabetes of the exocrine pancreas: American Diabetes Association-compliant lexicon. Pancreatology 2017;17:523-6.

20. Shen HN, Yang CC, Chang YH, Lu CL, Li CY. Risk of diabetes mellitus after first-attack acute pancreatitis: a national population-based study. Am J Gastroenterol 2015;110:1698-706.

21. Chand SK, Pendharkar SA, Bharmal SH, Bartlett AS, Pandol SJ, Petrov MS. Frequency and risk factors for liver disease following pancreatitis: a population-based cohort study. Dig Liver Dis 2019;51:551-8.

22. Cho J, Walia M, Scragg R, Petrov MS. Frequency and risk factors for mental disorders following pancreatitis: a nationwide cohort study. Curr Med Res Opin 2019;35:1157-64.

23. Quan H, Sundararajan V, Halfon P, Fong A, Burnand B, Luthi JC, et al. Coding algorithms for defining comorbidities in ICD-9-CM and ICD-10 administrative data. Med Care 2005;43:1130-9.

24. Lee SH, Hsu TC, Lee MG, Chao CC, Lee WC, Lai CC, et al; National Taiwan University Health Economics and Outcome Research Group. Nationwide trend of sepsis: a comparison among octogenarians, elderly, and young adults. Crit Care Med 2018;46:926-34.

25. Fisher LD, Lin DY. Time-dependent covariates in the Cox proportional-hazards regression model. Annu Rev Public Health 1999;20:145-57.

26. Nath SD, Voruganti VS, Arar NH, Thameem F, Lopez-Alvarenga JC, Bauer R, et al. Genome scan for determinants of serum uric acid variability. J Am Soc Nephrol 2007;18:3156-63.

27. Wilk JB, Djousse L, Borecki I, Atwood LD, Hunt SC, Rich SS, et al. Segregation analysis of serum uric acid in the NHLBI family heart study. Hum Genet 2000;106:355-9.

28. Yang Q, Guo CY, Cupples LA, Levy D, Wilson PW, Fox CS. Genome-wide search for genes affecting serum uric acid levels: the Framingham heart study. Metabolism 2005;54:1435-41.

29. Tai V, Merriman TR, Dalbeth N. Genetic advances in gout: Potential applications in clinical practice. Curr Opin Rheumatol 2019; 31:144-51

30. Elizalde-Barrera CI, Estrada-Garcia T, Lozano-Nuevo JJ, Garro-Almendaro AK, Lopez-Saucedo C, Rubio-Guerra AF. Serum uric acid levels are associated with homeostasis model assessment in obese nondiabetic patients: HOMA and uric acid. Ther Adv Endocrinol Metab 2017;8:141-6.

31. Chou P, Lin KC, Lin HY, Tsai ST. Gender differences in the relationships of serum uric acid with fasting serum insulin and plasma glucose in patients without diabetes. J Rheumatol 2001;28:571-6.

32. Gillies NA, Pendharkar SA, Asrani VM, Mathew J, Windsor JA, Petrov MS. Interleukin-6 is associated with chronic hyperglycemia and insulin resistance in patients after acute pancreatitis. Pancreatology 2016;16:748-55.

33. Gillies NA, Pendharkar SA, Singh RG, Asrani VM, Petrov MS . Lipid metabolism in patients with chronic hyperglycemia after an episode of acute pancreatitis. Diabetes Metab Syndr 2017;11 Suppl 1:S233-41.

34. Pendharkar SA, Asrani VM, Xiao AY, Yoon HD, Murphy R, Windsor JA, et al. Relationship between pancreatic hormones and glucose metabolism: a cross-sectional study in patients after acute pancreatitis. Am J Physiol Gastrointest Liver Physiol 2016;311:G50-8

35. Pendharkar SA, Singh RG, Bharmal SH, Drury M, Petrov MS. Pancreatic hormone responses to mixed meal test in new-onset prediabetes/diabetes after non-necrotizing acute pancreatitis. J Clin Gastroenterol 2020;54:e11-20.

36. Pendharkar SA, Singh RG, Petrov MS. Pro-inflammatory cytokine-induced lipolysis after an episode of acute pancreatitis. Arch Physiol Biochem 2018;124:401-9.

37. Tsushima Y, Nishizawa H, Tochino Y, Nakatsuji H, Sekimoto R, Nagao H, et al. Uric acid secretion from adipose tissue and its increase in obesity. J Biol Chem 2013;288:27138-49.

38. Zhu Y, Hu Y, Huang T, Zhang Y, Li Z, Luo C, et al. High uric acid directly inhibits insulin signalling and induces insulin resistance. Biochem Biophys Res Commun 2014;447:707-14.

39. Takir M, Kostek O, Ozkok A, Elcioglu OC, Bakan A, Erek A, et al. Lowering uric acid with allopurinol improves insulin resistance and systemic inflammation in asymptomatic hyperuricemia. J Investig Med 2015;63:924-9.

40. Major TJ, Topless RK, Dalbeth N, Merriman TR. Evaluation of the diet wide contribution to serum urate levels: meta-analysis of population based cohorts. BMJ 2018;363:k3951.

41. Batt C, Phipps-Green AJ, Black MA, Cadzow M, Merriman ME, Topless R, et al. Sugar-sweetened beverage consumption: a risk factor for prevalent gout with SLC2A9 genotype-specific effects on serum urate and risk of gout. Ann Rheum Dis 2014;73:2101-6.

42. Rasheed H, Phipps-Green A, Topless R, Hollis-Moffatt JE, Hindmarsh JH, Franklin C, et al. Association of the lipoprotein receptor-related protein 2 gene with gout and non-additive interaction with alcohol consumption. Arthritis Res Ther 2013;15:R177.

43. Rasheed H, Stamp LK, Dalbeth N, Merriman TR. Interaction of the GCKR and A1CF loci with alcohol consumption to influence the risk of gout. Arthritis Res Ther 2017;19:161.

44. Tu HP, Chung CM, Min-Shan Ko A, Lee SS, Lai HM, Lee CH, et al. Additive composite ABCG2, SLC2A9 and SLC22A12 scores of high-risk alleles with alcohol use modulate gout risk. J Hum Genet 2016;61:803-10.

45. Hamajima N, Naito M, Okada R, Kawai S, Yin G, Morita E, et al. Significant interaction between LRP2 RS2544390 in intron 1 and alcohol drinking for serum uric acid levels among a Japanese population. Gene 2012;503:131-6. 